\title{
A STUDY OF RELATIONSHIP BETWEEN SERUIM ESR AND CRP IN PATIENTS WITH OSSIFICATION OF POSTERIOR LONGITUDINAL LIGAMENT
}

KEY WORDS:

\section{Dr. Jagadish $\mathbf{T}$}

Dr. Sushant Ghumare*

\section{Dr. Pravin} Deokate
Junior Resident-3, B.J. Government Medical College and Sassoon General Hospital , Pune, Maharashtra, India.

Assistant Professor, B.J. Government Medical College and Sassoon General Hospital, Pune, Maharashtra, India. *Corresponding Author

Associate Professor, B.J. Government Medical College and Sassoon General Hospital, Pune, Maharashtra, India.

Ossification of the posterior longitudinal ligament (OPLL) is seen most commonly in men, in the elderly, and in Asian population [1]. The disease usually starts with minimal or no symptoms, but some patients slowly progress to develop symptoms of myelopathy and radiculopathy. The present study aims to find out the relationship between serum CRP and E ESR levels in patients with OPLL and its relationship with radiological classification of OPLL. In this cross sectional correlation study we have included all patients fulfilling the inclusion criteria from the year July 2019 to December 2020 in tertiary care hospital. Our study comprising of total 50 patients will show the relationship between OPLL with level of I $\quad$ serum CRP and ESR (normal or raised).In our study it was found that majority of the patients were above 50 years of age with male predominance.In the present study it was found that the level of CRP was raised in $83.4 \%$ cases of continuous type, $100 \%$ cases of localised type, $69.2 \%$ cases of mixed type and $92.8 \%$ cases of segmental type. In the present study it was found that the level of ESR was raised in $83.4 \%$ cases of continuous type, $66.7 \%$ cases of localised type, $61.5 \%$ cases of mixed type and $75.0 \%$ cases of segmental type. It is possible that raised serum levels of CRP and ESR may be associated with OPLL and thus inflammation may be the cause of OPLL.

\section{INTRODUCTION}

Ossification of the posterior longitudinal ligament (OPLL) is characterized by formation of ectopic new bone that serves as the replacement for ligamentous tissue (Fig l) [1,2]. OPLL can cause narrowing of the spinal canal and thus it has been recognized as one of the major causes of neurological impairment, like cervical myelopathy and/or radiculopathy $[2,3]$. This disease was thought to occur more frequently in Asians, but now, OPLL is known to occur in Caucasians also [4]. An accurate diagnosis through the use of plain radiograph, computed tomography (CT) and magnetic resonance imaging (MRI)findings is very important to monitor the development of symptoms and to make decisions regarding a treatment plan. When symptoms are mild and nonprogressive, conservative treatments and periodic observations are good enough, but once symptoms of myelopathy are present and neurologic symptoms are progressive, the treatment of choice is surgery to relieve spinal cord compression. It has been reported that several biomarkers, such as leptin and insulin, are related to OPLL $[5,6]$. However, we do not know whether or not inflammation occurs in OPLL. A previous study demonstrated that the Creactive protein (CRP) level is increased in patients with heterotopic ossification after total hip replacement [7]. The present study was prospectively designed to determine whether or not the serum CRP and erythrocyte sedimentation rate (ESR)concentration are altered in patients with OPLL so that if there is raised level of serum CRP and ESR is found in patients with OPLL it might be possible that inflammation is one of the causes of OPLL and thus anti-inflammatory therapy might be useful in these patients.

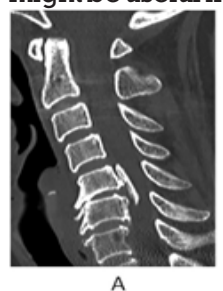

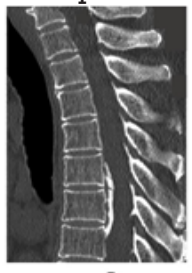

B

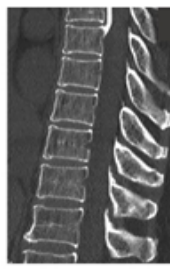

C

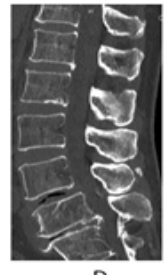

D
Fig 1. CT images of a patient with ossification of the posterior longitudinal ligament (OPLL). OPLL at C5, 5-6, 6 (A), T5, 5-6, 6 6-7, 7 (B, C) Ll-2 and L5-S1 (D).
Classifications Based On Lateral Radiograph Or Sagittal CT Images :

The Investigation Committee for Ossification of the Spinal Ligaments (part of the Japanese Ministry of Health, Labor and Welfare) developed a classification system for cervical OPLL comprising 4 types: [8]

(1) Continuous:a long lesion extending over several vertebral bodies;

(2) Segmental: one or several separate lesions behind the vertebral bodies;

(3) Mixed: a combination of the continuous and segmental types; and

(4) Localized (focal or circumscribed): located mainly just behind a disc space, ossification surrounding intervertebral disc herniation.

The above classification system is the most common system used in classifying OPLL.

\section{METHODOLOGY}

Ethics Approval:

A prospective study conducted at a tertiary care centre of B.J. Medical College and Sassoon Hospital, Pune and approved by the regional ethical committee.

\section{Study Area:}

Department of Orthopaedics, B.J. Govt. medical college \& Sassoon General Hospital, Pune.

\section{Sample Size :}

All patients fulfilling the inclusion criteria from the year July 2019 to December 2020.

\section{Inclusion Criteria:}

All patients with radiologically diagnosed cases of OPLL

\section{Exclusion Criteria:}

- All patients with other degenerative spinal disorders like osteophytes and disc degeneration and prolapse.

- Patients with diagnosed neurological dysfunction such as-

a. Stroke leading to monoparesis / monoplegia / paraparesis/ paraplegia / quadriparesis / quadriplegia 
b. Peripheral neuropathies

- Patients with traumatic cervical spine injury.

- Patients with congenital spine deformities.

- Patients with fractures in upper or lower limb.

- Tuberculosis of spine and other infections of spinal cord.

- Patients with systemic inflammatory disorders like Rheumatoid arthritis, Systemic lupus erythematosus, Multiple sclerosis.

- Patients with acute and chronic infections.

- Patients with liver disorders.

- Patients presenting with other conditions that may cause raise in ESR levels like anemia, kidney failure, pregnant women and obese.

- Patients presenting with polycythaemia, hyperviscosity, sickle cell anaemia, leukaemia, low plasma protein (due to liver or kidney disease) and congestive heart failure in whom ESR level may be decreased.

\section{Study Procedure:}

I. Detail history regarding onset and progression of symptoms.

II. History of trauma to be taken.

III. Any significant medical and surgical history.

IV.Detail neurological examination.

V. Radiograph- cervical / thoracic / lumbar spine based on history

VI. CT -- cervical / thoracic / lumbar spine based on history

VII. MRI spine-- cervical / thoracic / lumbar spine based on history

VIII. Lab investigations- Random blood sugar level,Hemoglobin, LFT, RFT, ESR, CRP

CRP is calculated using Latex slide agglutination method. (qualitative analysis)

Value $>0.6 \mathrm{mg} / \mathrm{dl}$ is considered as raised level .ESR is calculated using Westergren method.Value : Males $>15 \mathrm{~mm}$ in first hour is considered as raised.Females $>20 \mathrm{~mm}$ in first hour is considered as raised.

\section{OBSERVATIONS \& RESULTS}

- In our study it was found that majority of the patients were above 50 years of age accounting for $86 \%$ of the cases. Mean age of the study is $60.22+/-10.55$ years

- Male predominance was seen in the present study with $64 \%$ males to $36 \%$ females

- In the present study clinical features suggestive of myelopathy were found in $66 \%$ of cases and that of radiculopathy were found in $54 \%$ of patients.

- In the present study the vertebral level of involvement was maximum at the cervical spine (70\%) followed by thoracic (20\%) and lumbar (10\%) spine

\section{Distribution Of Study Group As PerThe Radiological Type Of OPLL}

In the present study the segmental type of OPLL was found to be most common type accounting for $56 \%$ followed by mixed type (26\%), continuous type (12\%) and localized type (6\%).

\section{Distribution Of Study Group As Per The Level Of Serum CRP And ESR}

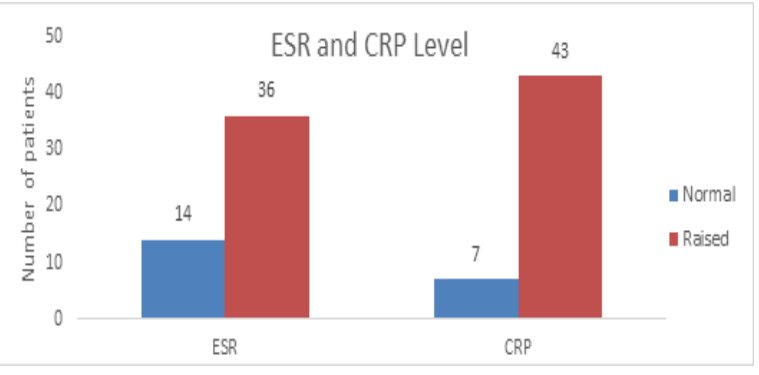

Fig: 2

|www.worldwidejournals.com $\mid$
In the present study it was found that ESR was raised in $72 \%$ of the cases while CRP was raised in $86 \%$ of the cases.

The confidence interval of proportion of raised ESR is 59.8 to 84.4 and that for CRP is 76.4 to 95.6

\section{Association Between Radiological Type Of OPLL And The CRP Levels}

In the present study it was found that the level of CRP was raised in $83.4 \%$ cases of continuous type, $100 \%$ cases of localised type, $69.2 \%$ cases of mixed type and $92.8 \%$ cases of segmental type. No significant correlation was found between the Radiological Type of OPLL and the level of CRP. [p-value > 0.05 (Not Significant) Chi-square test used]

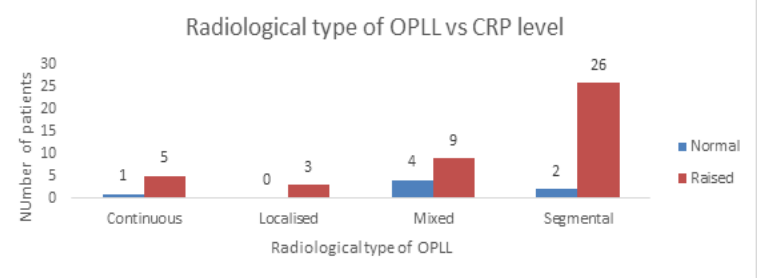

Fig: 3

Association between Radiological Type of OPLL and the ESR levels

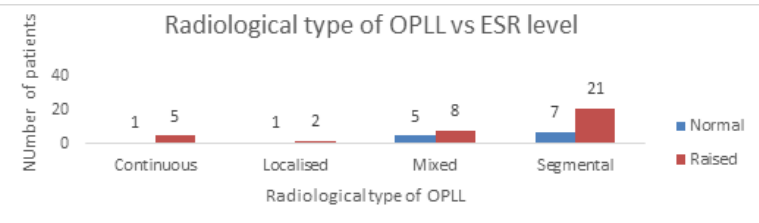

Fig: 4

In the present study it was found that the level of ESR was raised in $83.4 \%$ cases of continuous type, $66.7 \%$ cases of localised type, $61.5 \%$ cases of mixed type and $75.0 \%$ cases of segmental type. No significant correlation was found between the Radiological Type of OPLL and the level of ESR. [p-value > 0.05 (Not Significant) Chi-square test used.

-Data analysis performed by using SPSS (Statistical Package for social science) Version 25:0

-Qualitative data variables expressed by using frequency and Percentage (\%).

-Quantitative data variables expressed by using Mean, SD, Median etc.

-Chi-square test used to find the association between ESR level CRP levels with various qualitative data variables. $\mathrm{p}$-value $<0.05$ considered as significant.

\section{DISCUSSION}

A hospital based observational study was conducted to establish a relationship between serum CRP and ESR levels in patients with Ossification of Posterior Longitudinal Ligament (OPLL). Consecutive type of non-probability sampling was used for selection of cases. A total of 50 cases with OPLL, diagnosed on X ray, CT or MRI coming in Orthopaedics department OPD of a tertiary care hospital and fulfilling inclusion criteria were selected for study.In our present study it was found that of the total 50 patients with OPL, ESR was raised in $72 \%$ of the cases and it was normal in $28 \%$ of the cases. Similarly serum CRP level was raised in $86 \%$ of the cases and was normal in $14 \%$ of the cases.The confidence interval of proportion of raised ESR is 59.8 to 84.4 and that for CRP is 76.4 to 95.6 .

Similar results were also found in the study conducted by Yoshiharu Kawagachi et al.In their study on "Serum biomarkers in patients with ossification of the posterior longitudinal ligament (OPLL): Inflammation in OPL", they have found that of the 103 patients with OPLL and 95 age and sex matched volunteers with degenerative spinal disease in 
control group, the mean serum hs-CRP concentration was $0.122 \pm 0.141 \mathrm{mg} / \mathrm{dL}$ in the OPLL group and $0.086 \pm 0.114$ $\mathrm{mg} / \mathrm{dL}$ in the controls, producing a statistical difference between the two groups $(p=0.047)$. They also found that the ESR-lh and ESR-2h in the OPLL group were higher than those in the control group ( $p=0.003, p=0.002$, respectively). Based on this they concluded that inflammation can be one of the causes in the pathogenesis of OPLL. They also found a positive correlation between CRP and average length of OPLL progression per year [9].

Further in our study it was found that the level of CRP was raised in $83.4 \%$ cases of continuous type, $100 \%$ cases of localised type, $69.2 \%$ cases of mixed type and $92.8 \%$ cases of segmental type.

Also, the level of ESR was raised in $83.4 \%$ cases of continuous type, $66.7 \%$ cases of localised type, $61.5 \%$ cases of mixed type and $75.0 \%$ cases of segmental type.

No significant correlation was found between the radiological type of OPLL and the level of ESR and CRP.

\section{CONCLUSION}

In conclusion, the results observed in the study suggested that the level of ESR and serum CRP was raised in majority of the patients with OPLL. The association between radiological classification of OPLL and the level of ESR and CRP though was not statistically significant, it was raised in majority of patients of all types of OPLL. As ESR and CRP is raised in majority of the patients with OPLL and since the ESR and CRP being the markers of inflammation, it is possible that local inflammation may be involved in the pathogenesis of OPLL.

So if local inflammation is one of the key factors in the pathogenesis of OPLL then, it might be possible to prevent the progression of the OPLL by using simple anti-inflammatory drugs which is very cost effective.

A positive association is present between advancing age and the extent of OPLL.

The patients with OPLL can present with variable combination of features of myelopathy and radiculopathy without much significant difference between the incidence of myelopathy and radiculopathy.

\section{REFERENCES}

1. Byung-Wan Choi, K.-J. S. (2011). Ossification of the Posterior Longitudinal Ligament:A Review of Literature. Asian Spine JournalVol. 5, No. 4,267-276.

2. Tsukimoto H. On an autopsied case of compression myelopathy with a callus formation in the cervical spinal canal. Nihon-Geka-Hokan. 1960;29:1003-7.

3. Onji Y, Akiyama H, Shimomura Y, Ono K, Hukuda S, Mizuno S. Posterior paravertebral ossification causing cervical myelopathy.J Bone Joint Surg Am. 1967;49:1314-28.

4. Epstein NE. The surgical management of ossification of the posterior longitudinal ligament in 43 North Americans. Spine. (Phila Pa 1976) 1994; 19(6):664-72.

5. Ikeda Y, Nakajima A, Aiba A, Koda M, Okawa A, Takahashi K, et al. Association between serum leptin and bone metabolic markers, and the development of heterotopic ossification of the spinal ligament in female patients with ossification of the posterior longitudinal ligament. Eur Spine J. 2011; 20:1450-8. Epub 2011 Jan 22.

6. Akune T, Ogata N, Seichi A, Ohnishi I, Nakamura K, Kawaguchi H. Insulin secretory response is positively associated with the extent of ossification of the posterior longitudinal ligament of the spine. J Bone Joint Surg Am. 2001; 83:1537-44.

7. Sell S, Schleh T. C-reactive protein as an early indicator of the formation of heterotopic ossifications after total hip replacement. Arch Orthop Trauma Surg. 1999; $119(3-4): 205-7$

8. Tsuyama N. Ossification of the posterior longitudinal ligament of the spine. ClinOrthopRelat Res 1984;(184):71-84.

9. KawaguchiY,Nakano M,Yasuda T, Seki S, Suzuki K, YaharaY, Makino H, Kitajima I, Kimura T. Serum biomarkers in patients with ossification of the posterior longitudinal ligament (OPLL): Inflammation in OPLL. PLoS One. 2017 May 3;12(5):e0174881. 